\title{
Research of Carbon Fibre Absorbing Material Based on Impedance Matching
}

\author{
Yehua Li*, Daqing Huang, Shan He, Hongfei Cheng, Youqiang Shi \\ Beijing Institute of Aeronautical Material, Beijing, China

\section{Email address:} \\ rongchuanligang@163.com (Yehua Li), hdqbiam@163.com (Daqing Huang), biamhs333@sina.com (Shan He), \\ chenghongfei1989@16.com (Hongfei Cheng),npusyq@163.com (Youqiang Shi) \\ ${ }^{*}$ Corresponding author
}

\section{To cite this article:}

Yehua Li, Daqing Huang, Shan He, Hongfei Cheng, Youqiang Shi. Research Of Carbon Fibre Absorbing Material Based On Impedance Matching. Science Discovery. Vol. 4, No. 5, 2016, pp. 324-329. doi: 10.11648/j.sd.20160405.22

Received: September 8, 2016; Accepted: November 1, 2016; Published: November 5, 2016

\begin{abstract}
The carbon fibre has a good characteristic of electrical conductivity. When cut short and dispersed, it reacts as electric dipole or harmonic oscillator to radar wave. This paper blends the short carbon fibre with ethoxyline resin, and makes films by spraying the blend on prepreg which made by glass fibre and resin, then measures the EM parameters with the free-space method, and designs the low reflectivity absorbing material from $8 \mathrm{GHz}$ to $18 \mathrm{GHz}$ by matching impedance and calculating the reflectivity of multilayer films. Adjusting the kinds and quantities of matching layers can shift the absorbing frequency bands, and become better absorbing respective in X-band and Ku-band. The absorbing material samples are made by vacuumizing and heating method, and their reflectivity correspond to the designed result. The designing method and absorbing material made in this paper can be referred in engineering application.
\end{abstract}

Keywords: Short Carbon Fiber, Free-Space Method, Impedance Matching

\section{基于阻抗匹配的碳纤维吸波材料研究}

李业华, 黄大庆，何山, 程红飞, 史有强

北京航空材料研究院, 北京, 中国

\section{邮箱}

rongchuanligang@163.com(李业华), hdqbiam@163.com(黄大庆), biamhs333@sina.com(何山), chenghongfei1989@16. com(程红飞), npusyq@163.com（史有强）

摘要: 碳纤维具有良好的导电性, 经过短切和分散处理对雷达波作用时成为偶极子或谐振子, 具有较好的吸波效果。 本文将短切碳纤维与环氧树脂混合喷涂在玻璃纤维布与树脂做成的预浸料上制成胶膜，使用自由空间法测量胶膜的电 磁参数，对胶膜进行多层阻抗匹配及反射率计算，设计出在 $8 \sim 18 \mathrm{GHz}$ 具有较低反射率的吸波材料。通过调整匹配层的 种类和数量使吸波材料的吸收频带偏移, 分别对X波段和Ku波段实现良好的吸收。使用抽真空热压成型的方法制成吸波 材料样品, 测试反射率与设计相符。本文中的设计方法和制作的吸波材料可作为相关工程应用的参考。

关键词：短切碳纤维，自由空间法，阻抗匹配 


\section{1. 引言}

随着雷达探测技术的发展, 越来越多的装备提出了雷 达隐身性能要求, 雷达隐身技术成为各国武器装备发展的 重点。雷达隐身技术包括外形隐身技术和雷达吸波材料隐 身技术, 外形隐身技术是通过对武器装备的外形设计减少 电磁波向发射方向反射的技术，雷达吸波材料隐身技术是 在武器装备的表面使用吸波材料吸收电磁波减弱反射的 技术 [1]。按照吸波材料的应用方式, 可分为涂覆型吸波 材料、贴片型吸波材料、结构型吸波材料等。其中结构型 吸波材料具有承载和减小雷达反射截面的双重功能, 既能 减轻结构重量又能提高隐身性能 [2], 并具有吸收频带可 设计性强的优点, 结构型吸波材料成为隐身技术发展的重 点。

碳纤维复合吸波材料是一种重要的结构型吸波材料, 已在武器装备中获得广泛应用, 美国的B-2战略轰炸机、 $\mathrm{F} 22$ 战斗机、 $\mathrm{YF}-22$ 战斗机、 $\mathrm{YF}-23$ 战斗机、法国的 $\mathrm{F}-1$ 战斗 机、苏联的米格29战斗机以及先进的巡航导弹等大量采用 了碳纤维结构吸波材料, 碳纤维复合吸波材料在发达国家 的应用已经比较成熟。

国内在碳纤维复合吸波材料方面开展了一系列的研 究工作。何燕飞等采用磁性微粉与碳纤维制作的三层吸波 体在8 18GHz频段可实现反射率 $<-8 \mathrm{~dB}[3]$ 。赵东林等以 螺旋形碳纤维作为吸收剂制备了Nomex蜂窝夹芯结构吸波 材料, 厚度为 $9.5 \mathrm{~mm}$, 在 $3.76 \sim 18 \mathrm{GHz}$ 频段的反射率 $<-10 \mathrm{~dB}$, 最大吸收峰值可达 $-21.62 \mathrm{~dB}[4]$ 。刘辉等以镀镍中间相沥 青基碳纤维作为吸收剂环氧树脂为基体制备了单层吸波 涂层, 涂层厚度为 $1.02 \mathrm{~mm}$ 时在 $15.4 \sim 18 \mathrm{GHz}$ 反射率 $<-10$ $\mathrm{dB}$, 最大吸收峰可达 $-20.74 \mathrm{~dB}[5]$; 王雯结合湿法纺丝工 艺将含有还原铁粉的PAN聚合液纺制成纤维制备碳基复合 纤维材料, 材料涂层厚度为 $1.3 \mathrm{~mm}$ 时 $6 \sim 13.2 \mathrm{GHz}$ 和 $13.9 \sim$ $18 \mathrm{GHz}$ 反射率 $<-10 \mathrm{~dB}$, 最大吸收峰值可达 $-21.1 \mathrm{~dB}[6]$ 。周 勇将碳纤维镀镍制成镀镍碳纤维/环氧树脂吸波材料, 当 碳纤维质量分数在 $0.86 \%$ 纤维间距为 $5 \mathrm{~mm}$ 时, $13.15 \sim 18 \mathrm{GHz}$ 频段反射率 $<-10 \mathrm{~dB}$, 加入磁性金属Co粉拓宽了吸收频带, 在 $7 \sim 9 \mathrm{GHz}$ 和 $12.4 \sim 18 \mathrm{GHz}$ 大部分频段反射率 $<-10 \mathrm{~dB}[7]$ 。 邹田春等人研究了活化碳纤维偶极阵列/环氧树脂复合材 料的吸波性能, 当碳纤维偶极平行于电场方向时, 复合材 料反射率 $<-10 \mathrm{~dB}$ 的频宽达到 $12.2 \mathrm{GHz}$, 反射率最低可 达 $-32 \mathrm{~dB}[8]$ 。邹田春还研究了长度为 $1 \sim 2 \mathrm{~mm}$ 的活性碳纤维 的介电特性, 通过阻抗匹配方法设计了四层活性碳纤维吸 波复合材料，在 $7 \sim 15 \mathrm{GHz}$ 反射率 $<-10 \mathrm{~dB}$, 最大反射可达 -39. $3 \mathrm{~dB}[9]$ 。谢炜等以中空多孔聚丙烯腈 (PAN) 碳纤维为 主要吸收剂制备了双层轻质雷达吸波材料, 并以羰基铁粉 作吸收剂的匹配层提高吸波性能, 在 $4 \sim 18 \mathrm{GHz}$ 频率范围内 反射率 $\leqslant-10 \mathrm{~dB}$ 的带宽达 $10.90 \mathrm{GHz}[10]$ 。赵乃勤等制备了 含有直立碳纤维 (VACFs) 和活性碳纤维毡 (IACFFSs) 的环 氧树脂吸波复合材料，当IACFFSs中的束间距离、束宽以 及VACFs 中纤维间距分别为 $10 \mathrm{~mm} 、 5 \mathrm{~mm}$ 和 $8 \mathrm{~mm}$ 时, 复合材料 在 $11.8 \sim 18 \mathrm{GHz}$ 频段内的反射率均小于 $-20 \mathrm{~dB}[11]$ 。贺龙 辉等采用 $2 \mathrm{~mm}$ 与 $4 \mathrm{~mm}$ 两种短切碳纤维以 $3: 4$ 比例混合作为吸 收剂, 质量分数为 $7 \%$, 制备聚氨酯硬质泡沫基复合吸波 材料, 并以 $3 \%$ 填充量的多晶铁纤维加入聚氨酯基体中制
成厚度为 $3 \mathrm{~mm}$ 的匹配层, 总厚度为 $10 \mathrm{~mm}$, 在 $8 \sim 18 \mathrm{GHz}$ 频段 反射率可 $<-10 \mathrm{~dB}[12]$ 。

经过对碳纤维复合吸波材料的相关文献研究发现: (1) 单层碳纤维复合吸波材料的吸收频带比较窄, 反射率整体 偏高; (2) 多层碳纤维复合吸波材料中多数匹配层使用 的是磁性吸收剂, 制备工艺相对复杂, 并且磁性吸收剂对 温度以及环境比较敏感, 吸波性能容易变化; (3) 碳纤 维复合吸波材料受碳纤维排列方式影响很大, 对电磁波的 入射方向非常敏感。

吸波材料设计中为展宽吸收频带和降低反射率通常 采用多层阻抗匹配设计实现。本文对多层阻抗渐变设计原 理进行了分析, 采用阻抗渐变结构设计了 $8 \sim 18 \mathrm{GHz}$ 的多层 短切碳纤维树脂基复合吸波材料, 通过调整匹配层的种类 与数量改变了吸收频带和端点处的反射率值。试验中选用 短切碳纤维作为吸收剂, 环氧树脂作为载体, 喷涂在玻璃 纤维布与环氧树脂做成的预浸料表面, 制成碳纤维分布均 匀而含量不同的吸波胶膜, 使用自由空间法测量吸波胶膜 的电磁参数, 以此计算反射率和优化阻抗匹配, 设计了三 种不同吸收特性的吸波材料, 通过实验制备了样品测试了 反射率, 得到的测试结果与设计相符, 本文中的设计与研 制方法可以作为相关研究的参考。

\section{2. 实验材料及方法}

\section{1. 多层吸波材料设计原理}

多层吸波材料设计遵循微波传输线理论, 要实现良好 的吸收性能要求具备两个特点:（1）吸波材料表面阻抗 与空气阻抗接近, 减少入射电磁波在材料表面的反射; (2) 内层吸波材料对进入的电磁波实现高效损耗吸收, 减少电 磁波在底面发生反射后再次传出吸波材料。常见的阻抗匹 配结构有两种, 一种是阻抗渐变型, 即沿着电磁波入射方 向阻抗依次增大; 另一种是阻抗更迭型, 即沿着电磁波入 射方向在某些阻抗层后边插入阻抗更低的匹配层 [13]。本 文选用的是阻抗渐变型结构。

对于多层吸波材料, 结构如图1所示, 电磁波垂直入 射到多层吸波材料表面时, 各层的输入阻抗如（1）、（2） 式表示 [14]:

$$
\begin{gathered}
Z_{i 1}=\eta_{1} \tanh \left(\mathrm{k}_{1} \mathrm{~d}_{1}\right) \\
Z_{i k}=Z_{k} \frac{Z_{i(k-1)}+Z_{k} \tanh \left(\gamma_{k} d_{k}\right)}{Z_{k}+Z_{i(k-1)} \tanh \left(\gamma_{k} d_{k}\right)}
\end{gathered}
$$

式中 $Z_{k}$ 一第 $\mathrm{k}$ 层的特性阻抗,

$$
Z_{k}=\sqrt{\frac{\mu_{k}}{\varepsilon_{k}}}
$$

$\gamma_{k}$ 一第 $\mathrm{k}$ 层的传播常数, $\gamma_{k}=j \frac{\omega}{c} \sqrt{\mu_{k} \varepsilon_{k}}$;

$d_{k}$ 一第 $\mathrm{k}$ 层的介质厚度;

$\mu_{k}$ 一第 $\mathrm{k}$ 层的相对磁导率; 
$\varepsilon_{k}$ 一第k层的相对介电常数;

在吸波材料表面反射系数为:

$$
\Gamma=\frac{Z_{\text {in }}-Z_{0}}{Z_{\text {in }}+Z_{0}}
$$

反射率以 $d B$ 为单位表示为:

$$
\Gamma_{\mathrm{dB}}=20 \lg |\Gamma|
$$

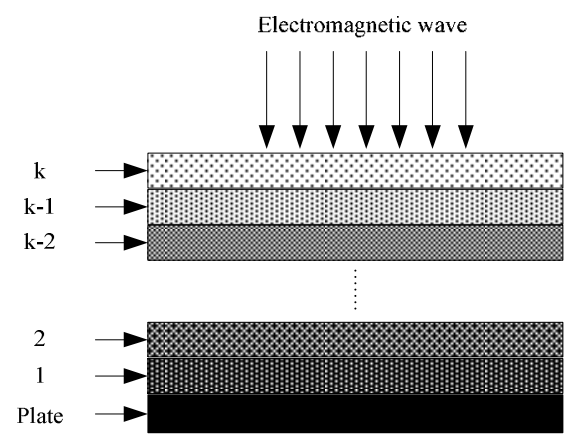

图1 多层吸波材料结构示意图。

\section{2. 实验选材}

碳纤维由石墨结晶沿纤维轴向排列而成, 含碳量在 $90 \%$ 以上，属于电损耗型吸波材料，具备耐高温、耐磨、耐腐 蚀、导电导热以及柔软各向异性等特点。目前对碳纤维吸 波机理的解释有两种: 一是碳纤维在入射电磁波激励下 发生谐振, 形成耗散电流, 耗散电流以热能为主要方式被 基体材料等逐渐消耗; 二是碳纤维在入射电磁波激励下 发生极化, 以偶极子的形式产生极化耗散电流, 在周围基 体材料作用下电磁能转换其它形式的能量衰减掉 [15]。碳 纤维的长度以及含量直接影响着吸波材料的电磁参数, 并 且随着入射电磁波的频率而改变, 具有频响特性。以短切 碳纤维与环氧树脂制作的碳纤维复合材料样品在碳纤维 含量为 $1 \mathrm{wt} \%$ 时, $4 \mathrm{~mm}$ 长的碳纤维具有较大的复介电实部和 虚步, 并具有良好的频响特性 [16]。在实验中以喷涂方式 制作的碳纤维吸波材料时碳纤维湿重百分比一般不能超 过 $1.5 \mathrm{wt} \%$, 若超过 $1.5 \mathrm{wt}$ \% 则容易因团聚堵塞管道。常用的 喷枪口径一般不超过 $4 \mathrm{~mm}$, 对于 $4 \mathrm{~mm}$ 长的短切碳纤维适用的 喷枪口径为 $3 \sim 4 \mathrm{~mm}$ 。本文中选用的碳纤维为 $T 700$, 长度为 $4 \mathrm{~mm}$ 。本文实验中使用的原材料如表 1 所示。

表1 实验原材料。

\begin{tabular}{lll}
\hline 原材料名称 & 型号 & 生产厂家 \\
\hline 短切碳纤维 & $\mathrm{T} 700,4 \mathrm{~mm}$ & 上海骐杰碳素材料有限公司 \\
玻璃纤维布 & $\mathrm{EW}-100 \mathrm{a}$ & 湖北菲利华石英玻璃股份有限公司 \\
环氧树脂 & $\mathrm{ME} 301$ & 北京吴天嘉诚科技有限公司 \\
环氧树脂 & $\mathrm{E} 44$ & 无锡钱广化工原料有限公司 \\
固化剂 & $\mathrm{D} 400$ & 常州市润翔化工有限公司 \\
二甲苯 & 分析纯 & 国药集团试剂有限公司 \\
丙酩 & 分析纯 & 国药集团试剂有限公司 \\
\hline
\end{tabular}

\section{3. 原材料处理}

将EW-100a玻璃纤维布与ME301环氧树脂通过热熔法 制成预浸料。碳纤维表面有层胶体, 需将胶体去除使碳纤 维分散, 避免团聚。称取适量短切碳纤维放置于烧杯中, 往烧杯中加入丙酮漫过碳纤维浸泡, 将烧杯放入超声波清 洗机中清洗分散30min, 清洗后将烧杯中的丙酮倒出, 将 碳纤维烘干, 对短切碳纤维电镜扫描如图2所示, 可见在 清洗之后碳纤维表面无杂质。称取晾干后不同量的短切碳 纤维分别与E44环氧树脂、D400固化剂、二甲苯、丙酮按 照一定比例混合均匀, 制成5种碳纤维含量的混合溶液。 将 5 种溶液按照一定量均匀喷涂在预浸料表面晾干, 制成5 种不同碳纤维含量的吸波胶膜, 编号为 A、B、C、D、E。 将喷涂C胶膜的混合溶液喷涂在脱膜纸上，如图3所示，可 见碳纤维分布均匀, 可避免短切碳纤维因取向不同而导致 电性能的差异 [17]。

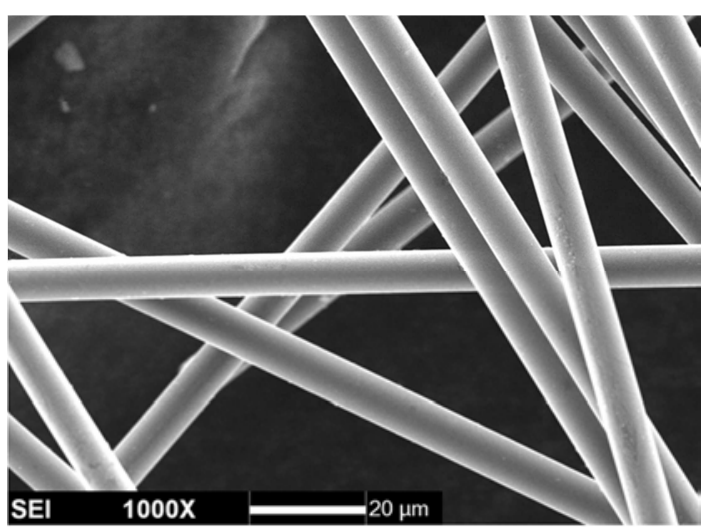

图2 短切碳纤维电镜扫描图。

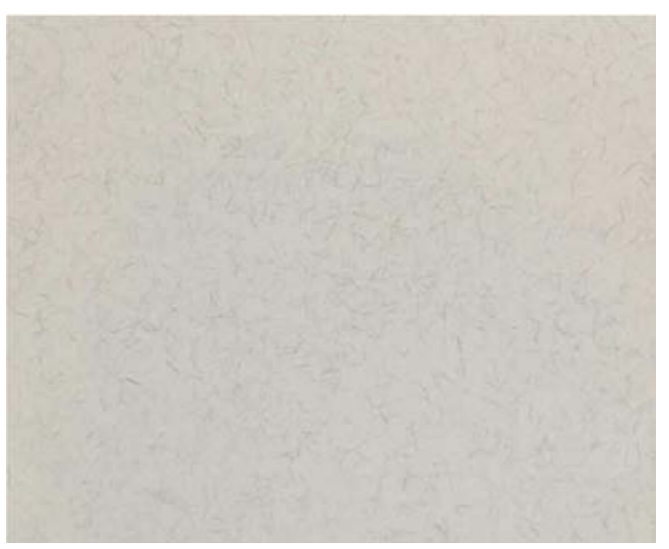

图3 短切碳纤维喷涂分布图。

\section{4. 电磁参数测量}

材料电磁参数的测量方法有多种, 包括驻波法、谐振 腔法、传输/反射法、自由空间法等，对于损耗超过 0.1 的大损耗材料, 电磁参数测量方法主要使用同轴法、波导 法和自由空间法, 其中同轴法使用的频率范围为 $1 \sim 18 \mathrm{GHz}$, 波导法和自由空间法的适用范围为 $2.6 \sim 40 \mathrm{GHz}$ 。近几年随 着矢量网络分析仪的更新, 自由空间法的测试频率范围已 经拓展到 $75 \sim 110 \mathrm{GHz}$ 。

自由空间法是一种非接触和非破坏性的测试方法, 对 测试样品没有严格的形状和工艺要求, 只需厚度均匀且有 
足够大的测试面积, 使电磁波均匀照射到样品表面并避免 边缘绕射即可 [18]。

在以往工程应用中由于测试技术及设备限制，通常对 粉末状吸收剂或一定厚度的吸波材料样品分别采用同轴 法和波导法测量电磁参数作为评估和计算使用。而在吸波 材料对电磁波的吸收损耗中, 除了吸收剂对电磁波的损耗, 吸收剂载体亦对电磁波产生作用, 载体的电磁参数影响着 电磁波的反射、透波以及热损耗性能。吸收剂与载体对电 磁波的综合作用效果才是真实的吸波效果。使用自由空间 法测量吸波材料样品可以得到吸波材料整体的电磁参数, 对于计算反射率设计隐身性能更准确。

本文中使用自由空间法对5种碳纤维含量的吸波胶膜 测量电磁参数, 测试系统的矢量网络分析仪型号为安捷伦 $\mathrm{E} 8363 \mathrm{~B}$, 测试软件为安捷伦 $85071 \mathrm{E}$ 。测量5种胶膜电磁参 数发现磁损耗非常小，因而更换为介电常数测量。对测试 数据进行平滑处理得到的电导率实部和虚部如图4、5所示。

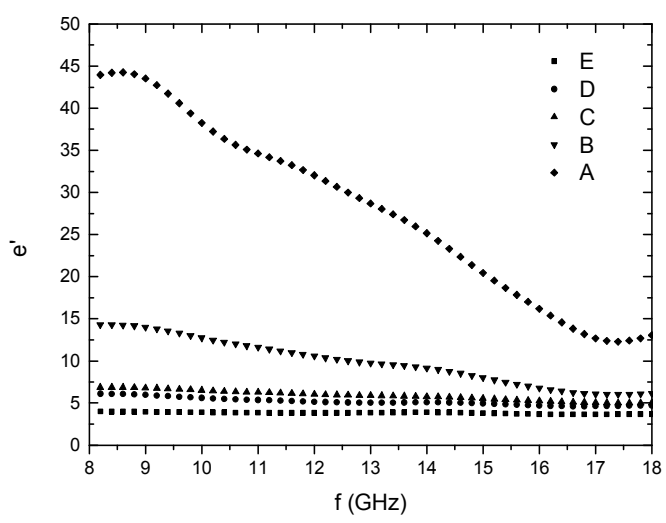

图4 5种胶膜的电导率实部。

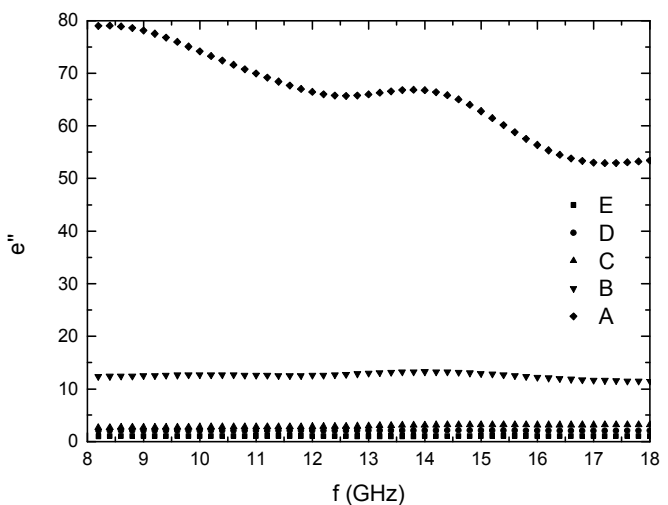

图5 5种胶膜的电导率虚部。

\section{5. 吸波材料设计}

依据阻抗斩变原理, 对含有短切碳纤维的吸波胶膜进 行多层设计, 按照电导率从大到小的顺序将胶膜A、B、C、 D、E从下往上依次排列层叠, 将5种吸波胶膜按照表 2 中顺 序和数量层叠得到1\#、2\#、3\#吸波材料样品。1\#样品使用
3 种胶膜, $2 \# 、 3 \#$ 样品与 $1 \#$ 样品相比在吸波材料内部增加 了中间梯度层，调整了吸波胶膜的数量，改变了吸波材料 内部的阻抗匹配。使用 5 种吸波胶膜的电磁参数计算得到 3 种吸波材料样品的反射率和输入阻抗的阻抗圆图, 如图6、 7所示。

表2 吸波材料各层编号及数量。

\begin{tabular}{llllll}
\hline 胶膜编号 & $\mathrm{A}$ & $\mathrm{B}$ & $\mathrm{C}$ & $\mathrm{D}$ & $\mathrm{E}$ \\
\hline 厚度 $(\mathrm{mm})$ & 0.25 & 0.2 & 0.2 & 0.2 & 0.2 \\
1\#, 层数 & 8 & 0 & 8 & 0 & 8 \\
2\#, 层数 & 8 & 3 & 6 & 2 & 8 \\
3\#, 层数 & 5 & 2 & 5 & 2 & 5 \\
\hline
\end{tabular}

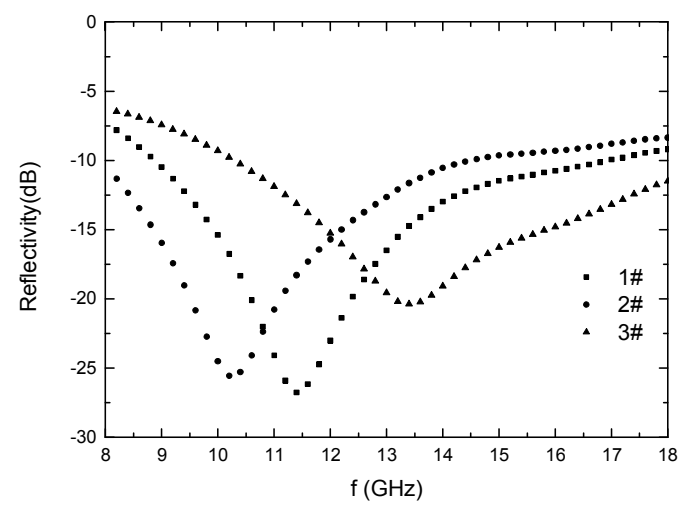

图6 吸波材料反射率计算结果。

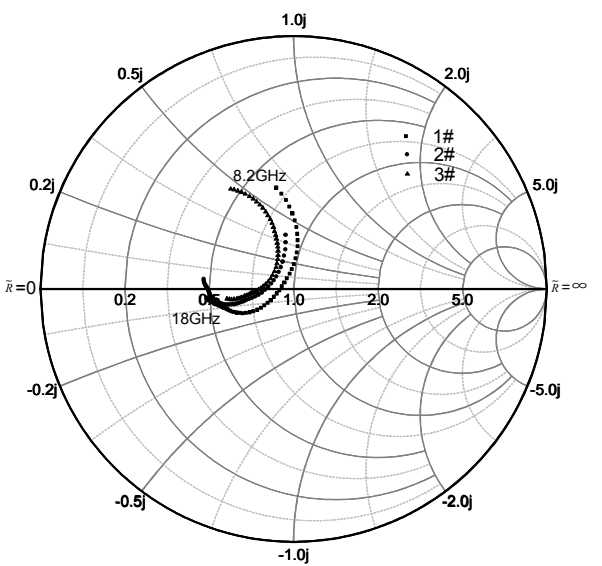

图7 吸波材料阻抗圆图。

\section{6. 样品制备与测试}

将A、B、C、D、E 5 种吸波胶膜裁剪成 $200 \mathrm{~mm} \times 200 \mathrm{~mm}$ 的方形胶膜, 按照表 2 中吸波材料样品的设计方案层叠, 使用真空袋将层叠好的吸波材料密封, 用真空泵连接真空 袋，抽压-0. $1 \mathrm{Mpa}$ ，将压紧的吸波材料放入烘箱在 $120^{\circ} \mathrm{C}$ 高 温下热压 $120 \mathrm{~min}$ 固化成型。冷却后从 $200 \mathrm{~mm} \times 200 \mathrm{~mm}$ 样品中 间裁剪出 $180 \mathrm{~mm} \times 180 \mathrm{~mm}$ 的测试样品, 厚度与重量如表 3 所 示。

按照GJB 2038A-2011标准中的弓形测试法测量反射 率, 如图8所示, 得到1\#、2\#、3\#样品的反射率曲线, 如 
图9所示。选取2\#样品顺时针旋转 $90^{\circ}$ 再次测试反射率, 对比旋转前后反射率如图10所示。

表3 吸波材料样品的厚度与重量。

\begin{tabular}{llll}
\hline 样品编号 & $1 \#$ & $2 \#$ & $3 \#$ \\
\hline 厚度 $(\mathrm{mm})$ & 5.10 & 6.03 & 3.92 \\
重量 $(\mathrm{g})$ & 212.17 & 236.94 & 166.25 \\
\hline
\end{tabular}

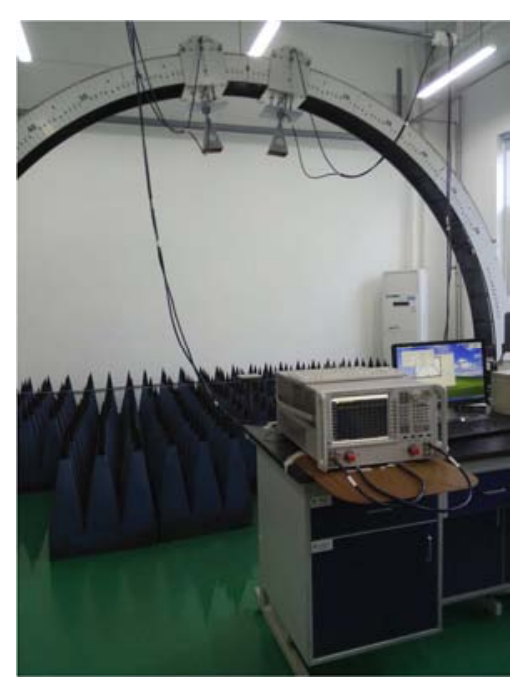

图8 吸波材料样品反射率测量图。

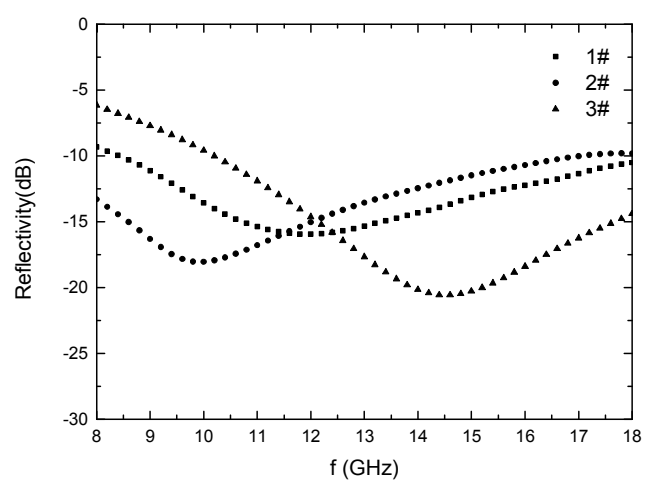

图9 吸波材料样品反射率测试结果。

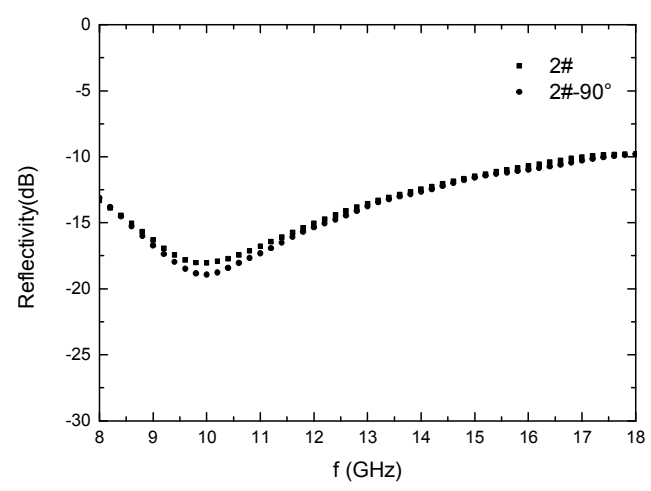

图102\#样品旋转 $90^{\circ}$ 反射率对比。

\section{3. 讨论}

由图6、9对比样品的反射率测试与计算结果可知, 1 \# 样品的 $-10 \mathrm{~dB}$ 吸收带宽增加 $1.6 \mathrm{GHz}$, 反射率在曲线两端下 降, 吸收峰向右偏移 $0.5 \mathrm{GHz} ; 2$ \#样品的 $-10 \mathrm{~dB}$ 吸收带宽增 大 $2.6 \mathrm{GHz}$, 反射率在曲线两端下降, 吸收峰向左偏移 $0.25 \mathrm{GHz} ; 3$ \#样品的 $-10 \mathrm{~dB}$ 吸收带宽基本不变, 反射率在曲 线右端下降, 吸收峰向右偏移 $1 \mathrm{GHz}$ 。对比可见, 三种设计 方案制备的吸波材料样品反射率与设计效果基本相符。

根据图7所示阻抗圆图分析样品的阻抗匹配情况, 输 入阻抗曲线经过阻抗圆图中的 $(1,0)$ 点时实现最佳阻抗 匹配, 入射到材料表面的电磁波被全部吸收没有反射。吸 波材料的输入阻抗随着频率变化, 在一个频段内输入阻抗 离（1，0）点越近匹配效果越好 [19]。图7中阻抗曲线对 应的频率上端为 $8.2 \mathrm{GHz}$ 下端为 $18 \mathrm{GHz}$, 频率从 $8.2 \mathrm{GHz}$ 增至 $18 \mathrm{GHz}$ 过程中, 1 \#样品的输入阻抗在中间频段离 $(1,0)$ 点近, 2 \#样品的输入阻抗在整个频段的前 $2 / 3$ 部分离 (1, 0) 点近, 而 3 \#样品的输入阻抗在整个频段的后 $2 / 3$ 部分离 ( 1,0$)$ 点近。3个样品在该频段的输入阻抗都是随频率 增加先接近 $(1,0)$ 点而后远离 $(1,0)$ 点, 匹配效果逐 渐变好再逐渐变差, 对应的反射率先降低后升高, 具有单 峰吸收特性。输入阻抗曲线多数位于 $\tilde{R}=0.5 、 \tilde{R}=1 、 \tilde{X}$ $=-0.2 j 、 \tilde{X}=0.5 \mathrm{j}$ 四条曲线围成的狭窄区域内, 输入阻抗 渐进变化分布密集, 说明样品的输入阻抗围绕最佳匹配点 变化较小，在整个频段具有良好的匹配特性。

对比 3 个样品使用的吸波胶膜分析反射率的变化, 1 \# 样品使用A、C、E 3种胶膜匹配实现了 8.4 18GHz反射率 $\leqslant-10 \mathrm{~dB}$ 的宽带吸收特性, 最低反射率为 $-15.95 \mathrm{~dB}$ 位于 $12 \mathrm{GHz}$ 。2\#与 1 \#样品的A、E胶膜数量相同, 2 \#样品在中间 减少了 $\mathrm{C}$ 胶膜的数量, 并增加少量的 $\mathrm{B} 、 \mathrm{D}$ 胶膜, 实现了 $8 \sim$ $17 \mathrm{GHz}$ 反射率 $\leqslant-10 \mathrm{~dB}$ 的宽带吸收特性, 最低反射率为 $-18.05 \mathrm{~dB}$, 位于 $9.9 \mathrm{GHz}$, 在 $8 \mathrm{GHz}$ 处反射率较 1 样品降低 $4 \mathrm{~dB}$, 相比吸收频带移向 $8.2 \sim 12.4 \mathrm{GHz}$ （X波段）。3\#与1\#样品 相比减少了 $\mathrm{A} 、 \mathrm{C} 、 \mathrm{E}$ 胶膜数量, 在中间增加少量的 B、D胶 膜, 3\#样品实现了 $10.2 \sim 18 \mathrm{GHz}$ 反射率 $\leqslant-10 \mathrm{~dB}$ 的宽带吸收 特性, 最低反射率为 $-20.58 \mathrm{~dB}$ 位于 $14.5 \mathrm{GHz}$, 在 $18 \mathrm{GHz}$ 处反 射率较1吸波材料降低 $3.9 \mathrm{~dB}$, 相比吸收频带移向 12.4 $18 \mathrm{GHz}$ （Ku波段）。

由图 10 可见选取的 2 \#吸波材料在旋转 $90^{\circ}$ 前后反射 率测试结果几乎完全一致, 说明碳纤维分散均匀使吸波材 料整体呈现各向同性特征。

综合以上讨论, 相比以往相关研究中出现的问题, 本 文使用的吸收剂单一, 制备工艺简单, 制作的吸波材料在 8 18GHz的-10dB吸收带宽相对更宽, 并且对电磁波的入 射方向不敏感, 便于应用。

\section{4. 结论}

（1）以短切碳纤维作为吸收剂制作的多层复合吸波 材料经过阻抗匹配设计可实现良好的宽带吸收 特性; 
（2）使用自由空间法测量的吸波胶膜电磁参数是吸 收剂与载体材料共同作用的结果, 以此计算的反 射率与样品测量的反射率具有很好的一致性;

（3）通过增加中间梯度层种类改变各梯度层的数量 可以调整阻抗匹配展宽吸收频带, 同时降低吸波 材料的厚度和重量;

（4）本文中制作的3种吸波材料样品在 $8 \sim 18 \mathrm{GHz}$ 的反 射率都比较低, 其中 2 \#样品在X波段性能较好, 3 \#样品在Ku波段性能较好, 并且制作的吸波材料 对方向不敏感, 可以用于工程应用以及研究参考。

\section{致谢}

本文为航空科学基金《新型宽带电损耗隐身结构复合 材料的力学建模和性能验证分析》(编号：20143121003) 的阶段性成果之一。

\section{参考文献}

[1] 田森. 复杂涂覆目标高频电磁散射特性研究 [D]. 南京: 南 京航空航天大学, 2011。

[2] 沈福贵, 逯贵祯, 梁晶晶. 宽带结构吸波材料特性研究 [J]. 微波学报：2012，（S3）：423-426。

[3] 何燕飞, 龚荣洲, 李享成, 王鲜, 何华辉. 多层复合吸波 材料的制备及其吸波性能 [J]。无机材料学报, 2006, 21 (6) : 1450-1453。

[4] 赵东林, 高云雷, 沈曾民. 螺旋形碳纤维结构吸波材料的制 备及其吸波性能研究 [J]. 功能材料信息, 2011, 8 (4) : 17-21。

[5] 刘辉, 高云雷, 赵东林, 迟伟东, 沈曾民. 镀镍中间相沥 青基碳纤维的吸波性能 [J].EMC材料应用，2011，（5）： 9-12。

[6] 王雯. 碳基复合吸波材料的制备及性能研究 [D]. 济南: 山 东大学，2012：89-90。
[7] 周勇. 镀镍碳纤维/环氧树脂复合材料的制备及吸波性能 研究 [D]. 武汉：武汉理工大学，2011：27-32。

[8] Zou T C, Shi C S, Zhao N Q. Microwave absorbing properties of activated carbon-fiber felt dipole array/epoxy resin composites[J]. Journal of Materials Science, 2007, 42(13) :4870-4876.

[9] 邹田春, 赵乃勤, 师春生. 活性碳纤维/树脂复合吸波材料 的设计 $[J]$. 功能材料与器件学报, $2007,13(1)$ ：54-58。

[10] 谢炜, 程海峰, 楚增勇, 陈朝辉, 周永江. 以中空多孔碳纤维 为主体的轻质吸波材料吸波性能研究 [J]. 无机材料学报, 2009, 24 (2) : 320-324。

[11] Zhao N Q, Zou T C, Shi C S, Li J J, Guo W W. Microwave absorbing properties of activated carbon-fiber felt screens (vertical-arranged carbon fibers)/epoxy resin composites $[\mathrm{J}]$. Materials Science and Engineering B, 2006, 127 (2) :207-211.

[12] 贺龙辉, 胡照文, 邓联文, 黄生祥, 刘胜, 贺君, 文瑞. 宽 频高性能短切碳纤维/聚氨酯泡沫吸波材料制备 [J]. 功能 材料，2015，23（46）：23120-23123。

[13] 齐宇, 黄大庆, 何山. 多层复合吸波涂料设计与试验研究 [J]. 航空材料学报：2010，30（2）：89-93。

[14] 马成勇, 程海峰, 唐耿平, 等. 三层雷达吸波涂层的吸波性 能研究 [J]. 材料工程：2008，（1）：11-13。

[15] 赵舟. 炭黑/碳纤维树脂基复合吸波材料性能研究 [D]. 海口: 海南大学, 2012: 35-39。

[16] 吴红焕. 短切碳纤维和炭黑的介电性能研究 [D]. 西安: 西北 工业大学, 2007：25-34。

[17] 郭伟凯. 碳纤维排布方式对结构吸波材料吸波性能的影响 及其机理分析 [D]. 天津:天津大学: 20-47。

[18] 张伟, 苏东林, 齐万泉. 基于自由空间法的复合材料电磁 参数测量 $[\mathrm{J}]$. 复合材料学报, 2007,24 (1)：141-145。

[19] 何山, 李业华, 周淳. 一种多层胶板雷达吸波材料 [J]. 航空 材料学报, 2016, 36 (4) : 41-46。 